\title{
Submillimetre Rectangular Waveguides based on SU-8 photoresist micromachining technology
}

\author{
David Glynn, Tianhao He, Jeff Powell, Yingtao Tian, Xiaobang Shang and Michael J. Lancaster \\ School of Electronic, Electrical and System Engineering \\ The University of Birmingham.
}

\begin{abstract}
Rectangular waveguides are fundamental structures for the transmission of signals at millimetre and submillimetre wavelengths. This paper describes the design and measured results for two rectangular waveguides based on layered SU-8 photoresist micromachining technology, with double-layer fabrication techniques to minimise the air gaps between layers. A brief description of the $\mathrm{SU}-8$ photoresist micromachining procedure is given in the paper. One waveguide is demonstrated for the WR-3 band from $220 \mathrm{GHz}$ to $325 \mathrm{GHz}$ the other is for the WR-6 band $120 \mathrm{GHz}$ to $170 \mathrm{GHz}$ both are made of layered SU-8 with a 3 piece construction. Both waveguides have novel bends in order to connect to the measurement apparatus. The measured performance is presented and compared to conventional machined metal waveguide structures. The measured insertion loss for the SU-8 waveguides in both bands is better than $0.03 \mathrm{~dB} / \mathrm{mm}$.
\end{abstract}

Keywords—waveguide; $\mathrm{SU}-8$; micromachining

\section{INTRODUCTION}

The desire for ever higher frequency communication systems has made implementing even the simplest of components difficult as terahertz frequencies are approached. However, due to micromachining technologies, rectangular waveguide transmission lines and passive circuits are now accessible for frequencies at submillimetre wavelengths. Such micromachined circuits are also ideal conduits for integrating active components and with novel methods of matching, efficient high frequency systems can be constructed. One such circuit considered when initiating this research is the frequency multiplier. Multipliers are used as it is difficult to generate a local oscillator frequency high enough to mix with a high frequency target signal. Frequency multipliers typically utilise a nonlinear harmonic response to distort a lower frequency into a required harmonic multiple. The frequency spans of the two waveguides in this paper were chosen to coincide with an input and doubled output frequency range for such a multiplying circuit. Care was taken to design the waveguides using just five layers of SU-8 photo resist, so that the waveguides orientations would allow an input circuit of $150 \mathrm{GHz}$ to connect to a future frequency doubler design, and the resultant $300 \mathrm{GHz}$ frequency would utilise the second waveguide. Ninety degree E- plane or and H-plane bends were constructed to connect to test equipment, or potentially to other stages in a communication system.

\section{WR-3 WAVEGUIDE DESIGN}

The rectangular waveguide designed here is a standard WR-3 band waveguide with a cross-section size of $a=864 \mu \mathrm{m}$ by $b=432 \mu \mathrm{m}$. The length of the waveguide is $15.95 \mathrm{~mm}$ to match previous waveguide circuits [1] and a novel bend is used to attach the waveguide to the measurement network analyser.

A device based on SU-8 pieces is easy to fabricate and is mechanically robust. It has a low insertion loss and good transmission coefficient for the specified operating frequency band. In this rectangular waveguide design, specifications for the transmission coefficient $S_{21}$ are better than $-20 \mathrm{~dB}$ for the whole WR-3 band. The WR-3 waveguide is built with 5 layers of SU-8 photoresist with each layer having a thickness of 288 $\mu \mathrm{m}$. Three layers form the total thickness of $864 \mu \mathrm{m}$ for the WR-3 waveguide internal dimensions with additional two layers for the top and bottom of the guide and to feed to the waveguide bends connecting the waveguide to the measurement apparatus as illustrated in Fig 1. The design of the waveguide bend is based on a similar previous structure in [1] and it is modified and configured to meet the dimension requirements of WR-3 waveguide. The waveguide bend is specified to be an H-plane.

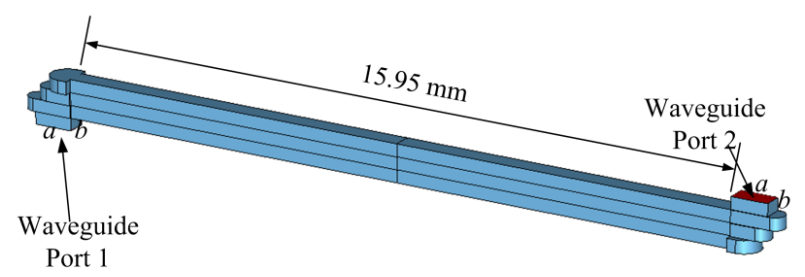

Fig. 1. 3D EM model for the WR-3 waveguide with bends. Here the inside of the metal waveguide is shown in blue and the layers are clearly seen.

\section{WR-6 WAVEGUIDE DESIGN}

The $150 \mathrm{GHz}$ waveguide dimensions are derived from the WR-6 waveguide specification $(a=1650 \mu \mathrm{m}, \quad b=830 \mu \mathrm{m})$, which covers the frequency range $110 \mathrm{GHz}$ to $170 \mathrm{GHz}$. WR-6 was chosen for its centre frequency of $150 \mathrm{GHz}$, which would be useful in a $300 \mathrm{GHz}$ communication system as part of the local oscillator multiplying circuit. However, because we are to 
construct the waveguide from layers of SU-8 photoresist which has a thickness of $288 \mu \mathrm{m}$ per layer, we are restricted to using a multiple of $288 \mu \mathrm{m}$ for one of its dimensions. The chosen implementation of the SU-8 photoresist layers is shown in Fig. 2 , and we can see with just 5 layers that a close approximation to WR- 6 can be made, where only the $b$ dimension differs in our solution, with an increase of $34 \mu \mathrm{m}(4 \%)$. A waveguide length of $15.95 \mathrm{~mm}$ was used.

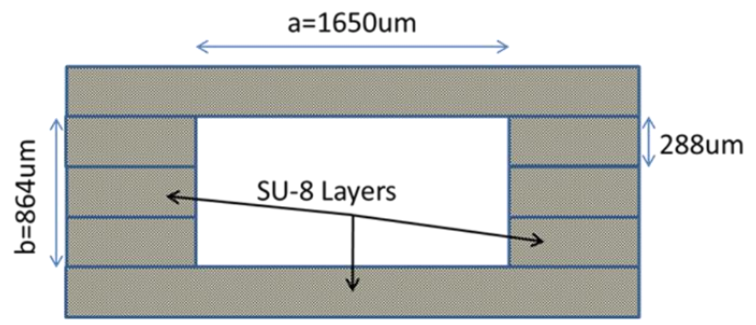

Fig. 2. Structure and Dimensions of $150 \mathrm{GHz}$ waveguide.

The $150 \mathrm{GHz}$ waveguide is to be constructed with 5 layers of SU-8 photoresist. Therefore the overall thickness of the device is $5 \times 288 \mu \mathrm{m}=1440 \mu \mathrm{m}$. Two ninety degree E-plane bends are needed to allow the connection of test equipment. Fig. 3 shows the configuration of the bends and the waveguide test ports perpendicular to the waveguide. There was also an additional complication in that only WR-5 $(a=1295.4 \mu \mathrm{m}$, $b=677.7 \mu \mathrm{m})$ test heads were available for the Vector Network Analyser, so a conversion was required for this within the bend structure.

The bend was designed by constructing a simple parameterised staircase structure which widens slightly from the WR-5 test head orifice to that shown in Fig. 2. A simple optimisation software routine was used to adjust each step to find the best solution

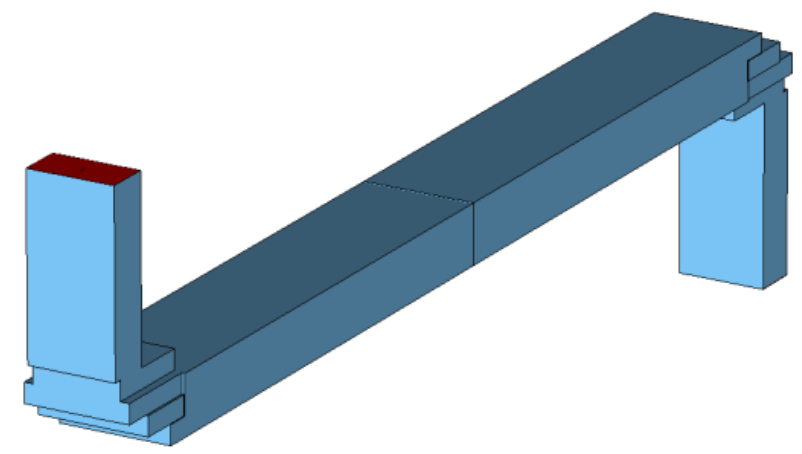

Fig. 3. 3D Model of $150 \mathrm{GHz}$ Waveguide and bends.

\section{FABRICATION}

Based on the CST waveguide bend model as shown in Fig. 1 , the layouts of the $5 \mathrm{SU}-8$ layers and their corresponding masks for micromachining photolithography are generated. There are five layers for the SU-8 waveguide and they are displayed in Fig. 4. As the whole structure is symmetrical to the centre point, the Layer 1 and Layer 5 are identical to the Layer 2 and Layer 4.

Both WR-3 and the WR-6 waveguides consist of 5 SU-8 layers, with each layer having a thickness of $288 \mu \mathrm{m}$. The fabrication integrates the top and bottom two layers with a joined double layer fabrication technique, thus making three standalone SU-8 pieces instead of 5 individual pieces. The top and bottom joined pieces are of same $576 \mu \mathrm{m}$ thickness and the middle piece is $288 \mu \mathrm{m}$. Thick SU-8 photoresist can be used as the structural material for constructing waveguides and is very cost effective, having a good fabrication repeatability and requires low facility investment in comparison with the precision $\mathrm{CNC}$ milling. All the procedures are performed in the in-house clean room facilities and the metallisation stage is completed in-house also. The fabrication process for the SU-8 pieces is detailed in [2].

Two waveguide circuits for the WR-3 band and one for the WR-6 band are fabricated, and a photo of the WR-3 waveguide circuit is shown in Fig. 5. The SU-8 waveguide device is fully metallised with silver on the surface of the SU-8 pieces. Each of the fabricated SU-8 pieces are assembled together with clamping brass plates reducing possible losses due to the air gaps. The main part of the photograph in Fig. 5 is the device clamped by brass plates occupies a volume of $48 \mathrm{~mm} \times 24 \mathrm{~mm}$ $\times 8 \mathrm{~mm}$.

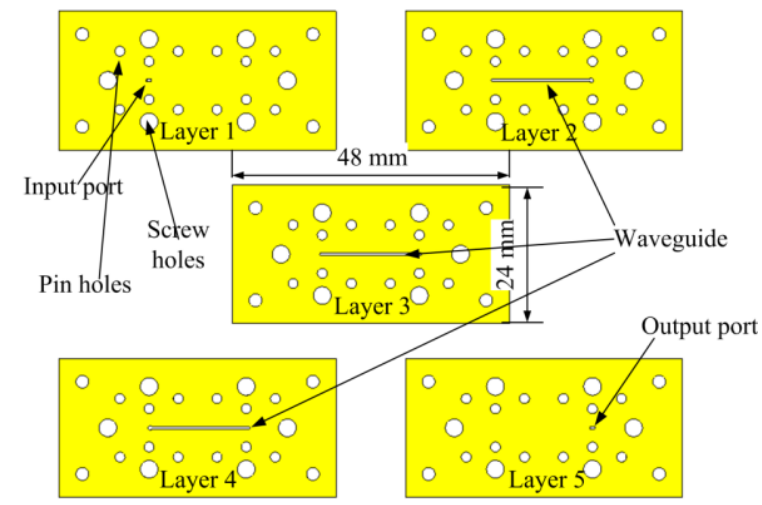

Fig. 4. The SU-8 layers for the WR-3 waveguide.

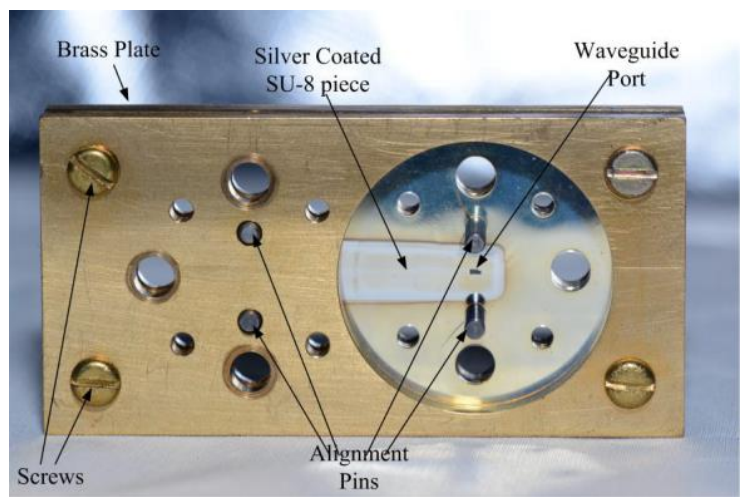

Fig. 5. The photograph shows a fabricated and assembled device WR-3 waveguide. 


\section{MEASUREMENTS}

Fig. 6 shows the measurement configuration of the fabricated and assembled WR-3 band waveguide circuit carried out on the Agilent E8361A Vector Network Analyser with a WR-3 OML band extender T/R module at test port 1 and a receiving only $\mathrm{T}$-module at port 2 . Enhanced response calibrations are done before the measurements. Standard UG387 waveguide flanges are used as the interfaces for the connection from WR-3 waveguide to the measurement ports. Screws and alignment pins are fastened to minimise the reflection, leakage and loss from the flanges.

\section{WR-3 WAVEGUIDE RESUlTS}

Two WR-3 band waveguide circuits are made in the inhouse clean room, and both have been tested and are illustrated in Fig. 7 for the insertion loss $S_{21}$ and in Fig. 8 for input return loss $\mathrm{S}_{11}$. One waveguide has the measured insertion loss of 2 $\mathrm{dB}$ to $1 \mathrm{~dB}$ over the tested frequency bandwidth from $220 \mathrm{GHz}$ to $325 \mathrm{GHz}$ and the other waveguide has the insertion loss of $0.5 \mathrm{~dB}$ over the main frequencies. Both measurements include the effect of the bends. In terms of the return loss, one waveguide is tested to have at least $15 \mathrm{~dB}$ over the frequency band from $220 \mathrm{GHz}$ to $310 \mathrm{GHz}$ and best of $20 \mathrm{~dB}$ at $300 \mathrm{GHz}$ while the other waveguide is tested to have average loss of 10 $\mathrm{dB}$ over the whole bandwidth and has the best response of 20 $\mathrm{dB}$ at $230 \mathrm{GHz}$.

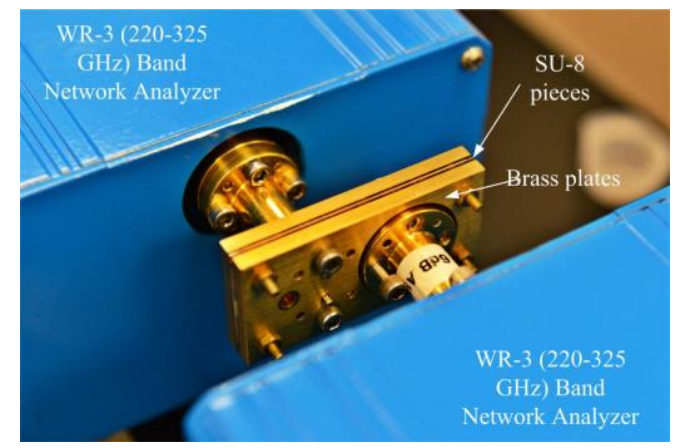

Fig. 6. The WR-3 waveguide under test with a Network Analyser.

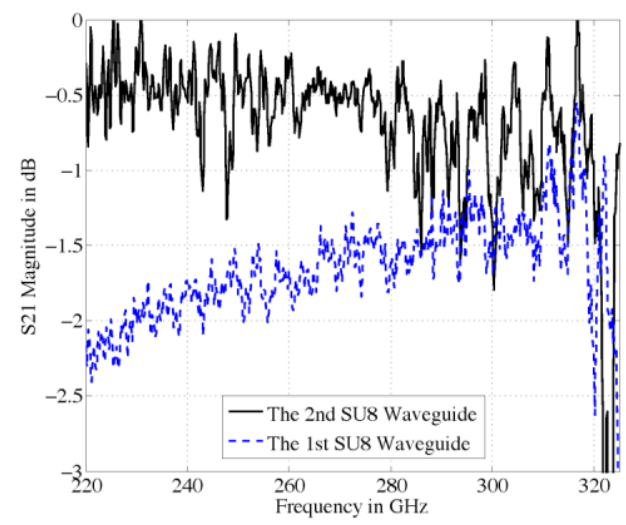

Fig. 7. The measurement results for the two fabricated results in $S_{21}$

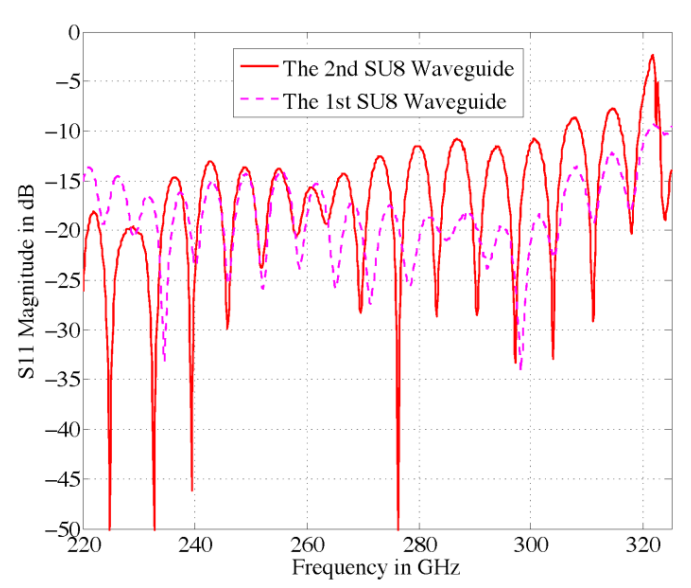

Fig. 8. The measurement results for the two fabricated results in $S_{11}$

\section{WR-6 WAVEGUIDE RESULTS}

The $150 \mathrm{GHz}$ waveguide was tested using the Agilent E8361A Vector Network Analyser with a pair of OML WR-5 heads (T/R module at test port 1 and a receiving only $\mathrm{T}$-module at port 2), covering the range from $140 \mathrm{GHz}$ to $220 \mathrm{GHz}$. The designed range is from $120 \mathrm{GHz}$ to $170 \mathrm{GHz}$, so we can only show measured results from $140 \mathrm{GHz}$ upwards, as shown in Fig. 9 and 10. The loss per $\mathrm{mm}$ is in shown in Fig. 11. $\mathrm{S}_{11}$ follows the simulated results well with a return loss better than $12 \mathrm{~dB}$ across the measured band, and an average return loss of $24 \mathrm{~dB}$. The $S_{21}$ result shows that the losses are better than 0.034 $\mathrm{dB} / \mathrm{mm}$ across the band, with an average loss of $0.011 \mathrm{~dB} / \mathrm{mm}$.

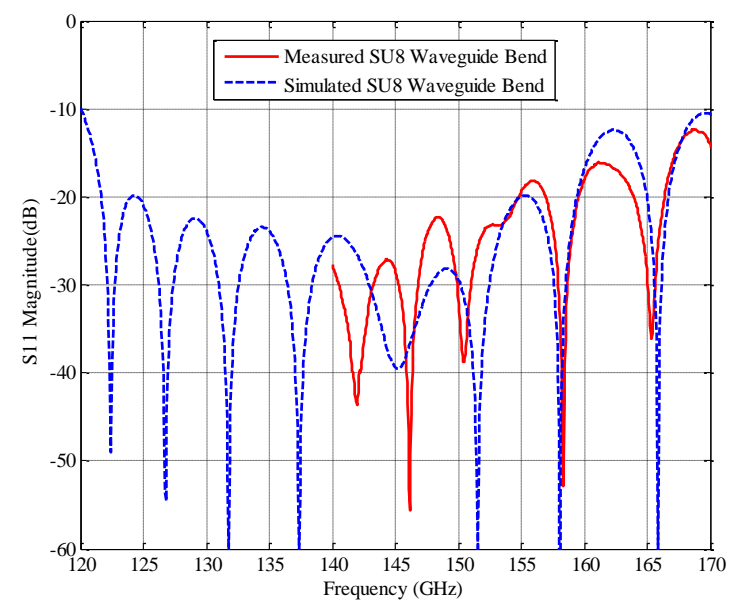

Fig. 9. $\mathrm{S}_{11}$ Results of $150 \mathrm{GHz}$ waveguide 


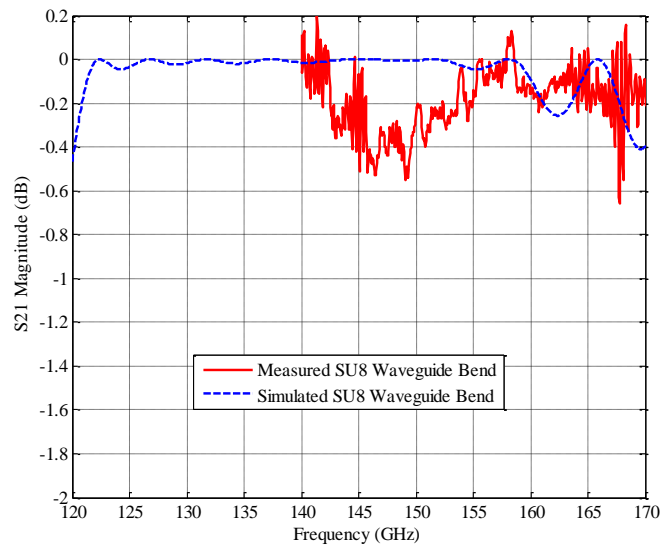

Fig. 10. $\mathrm{S}_{21}$ Results of $150 \mathrm{GHz}$ waveguide

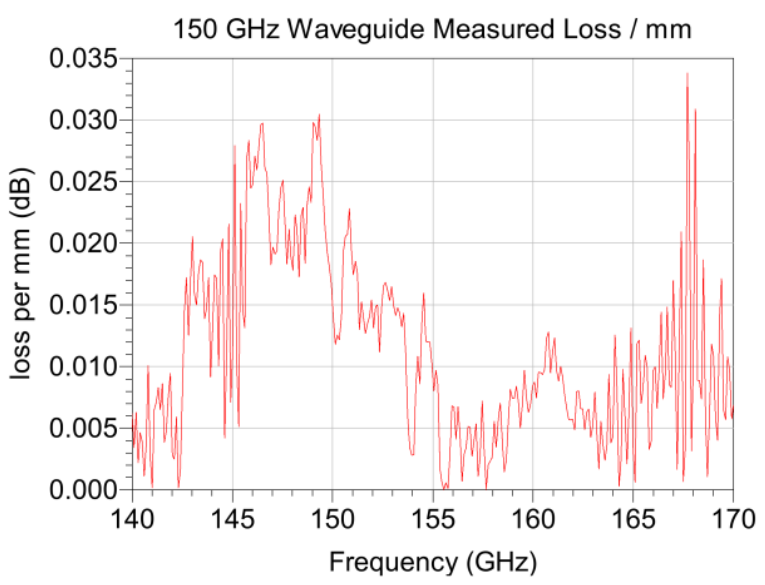

Fig. 11. Insertion Loss per mm for $150 \mathrm{GHz}$ waveguide

\section{CONCLUSION}

We have shown it is possible to design and fabricate two fit for purpose waveguides from 5 layers of SU-8 photoresist to operate at the WR-3 and WR-6 frequency bands, and also have good ninety degree bends to allow connection to test fixtures or other circuit stages. These would be ideal conduits for a future communication system or its sub circuits, such as a frequency multiplier.

\section{REFERENCES}

[1] X. Shang, ML Ke, Y. Wang, and MJ Lancaster. Micromachined wr-3 waveguide filter with embedded bends. Electronics Letters, 47(9):545547, 2011.

[2] Yingtao Tian, Xiaobang Shang, and Michael J. Lancaster. Fabrication of multi-layered su8 structure for terahertz waveguide with ultralow transmission loss. Journal of Micro/Nanolithography, MEMS, and MOEMS, 13(1):013002-013002, 2014.10.1117/1.JMM.13.1.013002. 